\title{
Un modèle de maladie de Huntington induite chez le rat par l'acide quinolinique
}

\section{Les nouvelles} de ce numéro ont été préparées par : J.-C. Dreyfus

P. Briand

A. Kahn S. Erlinger
La maladie de Huntington est une affection neurologique grave, qui se transmet comme un caractère autosomique dominant. Elle se caractérise par des mouvements choréiques accompagnant une détérioration mentale progressive. On en connaît la localisation génique (bras court du chromosome 4) mais on en ignore les mécanismes d'apparition et on ne possède aucun traitement. Les anomalies neurochimiques comportent une perte de certains neurotransmetteurs comme le GABA, alors que la dopamine n'est pas modifiée et la somatostatine est augmentée. On a cherché à reproduire ces anomalies expérimentalement. Un certain nombre d'agents dits " excitotoxiques " sont capables d'en provoquer, mais il affectent tous les types cellulaires sans manifester aucune sélectivité. En I983 Schwarcz et coll. [I] ont montré que l'acide quinolinique * (AQ), un métabolite du tryptophane, induit des lésions qui ressemblent à celles de la maladie de Huntington. Flint Beal et coll., de Boston [2], ont récemment apporté des précisions importantes. L'injection d'AQ dans le striatum de rats provoque une déplétion de GABA et de substance P, mais épargne électivement les neurones qui contiennent la somatostatine. Par contre, l'acide nicotinique, chimiquement proche de l'AQ ne déclenche aucun trouble.

Le fait que l'AQ reproduise les principales anomalies neurochimiques de la maladie de Huntington ne prouve évidemment pas qu'il en soit la cause in vivo. Cependant, il peut constituer la base d'une hypothèse de travail intéressante, en dirigeant les recherches vers un métabolisme anormal, excès de synthèse, insuffisance de dégradation ou sensibilité accrue à ce métabolite. On sait en outre que les concentrations en $A Q$ du cerveau sont faibles et ne sauraient entraîner une action toxique à court terme. Elles pourraient cependant suffire pour agir à long terme, et l'on sait que la maladie de Huntington ne se révèle cliniquement qu'à l'âge adulte et souvent tardivement. Mais l'intérêt le plus grand de cet abord est qu'il permet d'envisager pour la première fois une thérapeutique : la neurotoxicité de l'AQ pourrait être bloquée par des antagonistes [3], par exemple l'acide cynurénique.

J.-C. D.
- L'acide nicotinique (précurseur de la nicotinamide ou vitamine PP) et l'acide quinolinique sont deux métabolites du tryptophane produits par une voie différente de celle de la sérotonine. Ils ne diffèrent que par la présence d'un seul ou de deux groupements acides branchés sur le cycle pyridine.

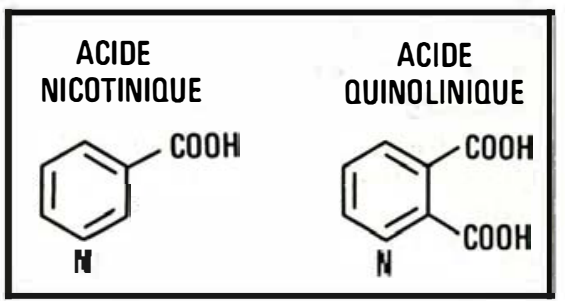

I. Schwarcz R, Whetsell WO, Mangano RM. Quinolinic acid: an endogenous metabolite that produces axon-sparing lesions in rat brain. Science $1983 ; 219: 316-9$.

2. Flint Beal M, Kovall MW, Ellison DW, et al. Replication of the neurochemical characteristics of Huntington's disease by quinolinic acid. Nature 1986; $321: 168-71$.

3. Schwarcz R, Meldrum B. Excitatory aminoacid antagonists provide a therapeutic approach to neurological disorders. Lancet 1985 ; ii : 140-3. 